\title{
Highly Efficient Synthesis of Hindered 3-Azoindoles via Metal-Free C-H Functionalization of Indoles
}

\author{
Nicolas Jacob \\ Lucas Guillemard \\ Joanna Wencel-Delord* \\ Laboratoire d'Innovation Moléculaire et Applications \\ (UMR CNRS 7042), Université de Strasbourg/Université de \\ Haute-Alsace, ECPM, 25 Rue Becquerel, 67087 Strasbourg, \\ France \\ wenceldelord@unistra.fr \\ Published as part of the Bürgenstock Special Section 2019 \\ Future Stars in Organic Chemistry
}

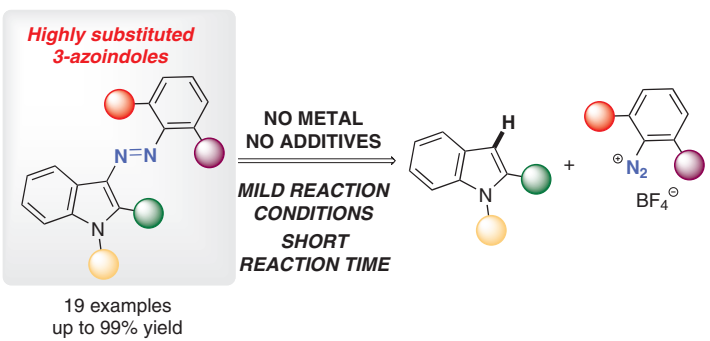

es will be selected (long thermal lifetime) while compounds characterized by short thermal lifetime are used in realtime optical information transmitting materials, ${ }^{10}$ in medicinal chemistry for neurons or ion channel stimulation purposes. ${ }^{11}$ Accordingly, several families of molecular photoswitches have been designed (Figure 1b) including spiropyranes, ${ }^{12}$ stilbenes ${ }^{13}$ and diarylethenes, ${ }^{14}$ but the azobenzenes ${ }^{15}$ are, by far, the most commonly applied ones (Figure 1a). More recently, indigoids ${ }^{16}$ or Stenhouse adducts ${ }^{17}$ have been disclosed. Considerable attention has also been focused on heteroazoswitches, ${ }^{18}$ including compounds featuring pyridine, imidazole, pyrazole, and purine motifs.

In clear contrast, 3-arylazoindoles are relatively underexplored molecules. ${ }^{19}$ Surprisingly, only few literature reports disclose synthesis of indoles bearing a diazo moiety in $\mathrm{C} 3$ position ${ }^{20}$ and the recent methodologies request use of sophisticated coupling partners such as aryltriazenes ${ }^{21}$ in ionic liquid medium or arylhydrazine hydrochlorides ${ }^{22}$ under visible-light irradiation or heating at $90^{\circ} \mathrm{C}$.

Very recently, a unique potential of 3-arylazoindole photoswitches has been demonstrated by König (Figure $1 \mathrm{c})^{23}$ and thus development of truly efficient, sustainable and straightforward protocols delivering such compounds is timely. In particular, as the properties of azoswitches, and especially their thermal lifetime, are impacted by the substitution pattern around the azo moiety, synthesis of a library of 3-arylazoindoles bearing various substituents in proximity of $\mathrm{N}=\mathrm{N}$ motif, on both $\mathrm{C} 2$ position of the indole and ortho-, ortho'-positions of the aromatic ring, seems very appealing (Figure 1d). ${ }^{24}$ Accordingly, we report herein an extremely simple but highly efficient strategy to prepare sterically hindered 2-substituted 3-arylazoindoles, the molecules with promising photochromic properties.

Our investigations began by exploring the coupling between 2-(tert-butyl)- $1 \mathrm{H}$-indole (1a) and electron-rich paramethoxyphenyldiazonium salt $\mathbf{2 a}$. The reaction occurred 


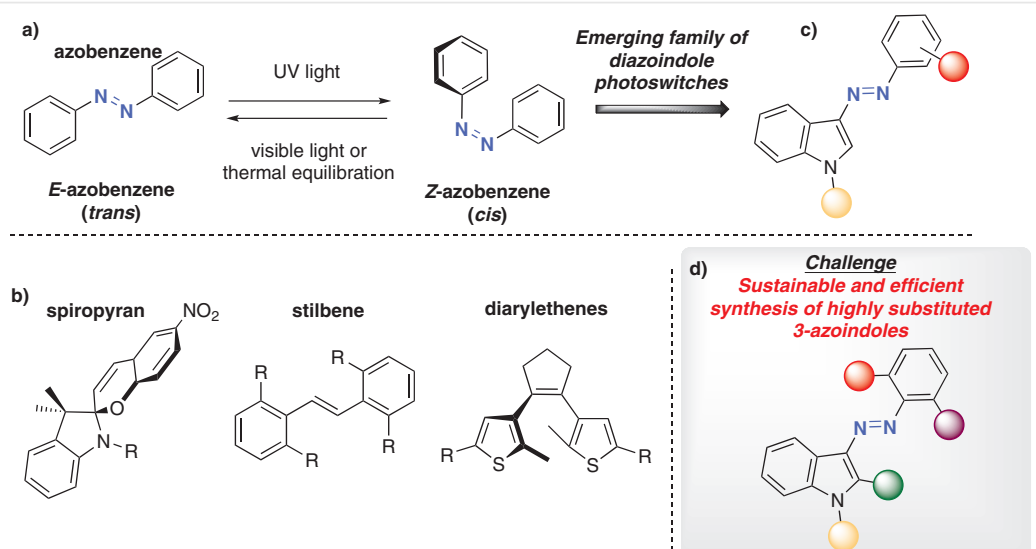

Figure 1 Several families of common photoswitch compounds

smoothly in methanol medium and at room temperature, delivering the expected (E)-2-(tert-butyl)-3-[(4-methoxyphenyl)diazenyl]-1H-indole (3a) in quantitative yield (Table 1 , entry 1 ). Comparable results were obtained when using 2-(methyl)- $1 H$-indole (1b) as substrate (entry 2 ). Besides, the reaction is extremely fast as full conversion of $\mathbf{1 b}$ could be achieved in less than 10 minutes (entry 3), even in the presence of equimolar amounts of both coupling partners (entry 4). Electron-poor Ac-substituted aryldiazonium salts may also be converted into 3-arylazoindoles, but the reaction generally requires a slight excess of the diazonium salts coupling partners (entry 5). Accordingly, the general reaction conditions have been determined, that is, use of 1.3 equivalents of diazonium salt in $\mathrm{MeOH}$ medium and 30 minutes as standard reaction time (entry 6). Of note is that the desired products are isolated via simple filtration of the crude mixture through silica gel pad, further demonstrating the experimental simplicity and efficiency of this protocol. This transformation hence perfectly follows the requirements of sustainable and green chemistry, as neither a catalysts nor sophisticated additives or strong oxidants are required and this coupling is characterized by excellent atom economy. Finally, the reaction performed in water is sluggish and the desired product $\mathbf{4 a}$ was formed in only $68 \%$ NMR conversion after 4 days (entry 7 ).

The generality of this new protocol was subsequently explored (Scheme 1). Rewardingly, indole 1a bearing a highly hindering tert-butyl motif in C2 position could be coupled very smoothly with diverse diazo coupling partners, both electron-rich and electron-poor, affording the expected products in excellent yields and in short reaction time. Importantly, the presence of a substituent in the ortho-position of $\mathbf{2}$ is tolerated well, as $\mathbf{3 b}, \mathbf{3} \mathbf{c}$, and $\mathbf{3 e}$ could be isolated in almost quantitative yields. In addition, very congested azoindole $\mathbf{3 g}$ could also be synthesized following the standard procedure in $93 \%$ yield. Interestingly, our protocol also tolerates relatively well a halogen atom on the indole scaffold, as $\mathbf{3 h}$ could be isolated in $70 \%$ yield, albeit excess of
Table 1 Optimization Study

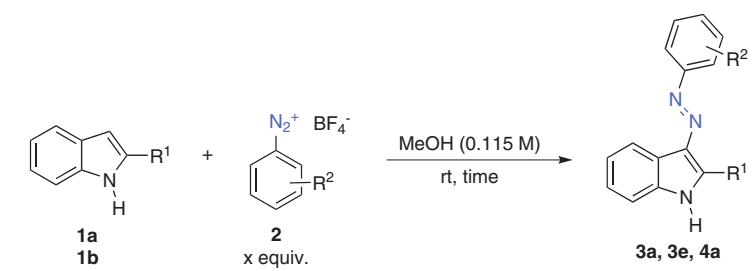

\begin{tabular}{|c|c|c|c|c|c|c|}
\hline Entry & $\mathrm{R}^{1}$ & $R^{2}$ & 3 & x (equiv.) & Time & Yield (\%) \\
\hline 1 & $t-B u$ & 4-OMe & $3 a$ & 1.5 & $16 \mathrm{~h}$ & 99 \\
\hline 2 & Me & 4-OMe & $4 a$ & 1.5 & $16 \mathrm{~h}$ & 99 \\
\hline 3 & Me & 4-OMe & $4 a$ & 1.5 & $10 \mathrm{~min}$ & 99 \\
\hline 4 & Me & 4-OMe & $4 a$ & 1.0 & $10 \mathrm{~min}$ & 99 \\
\hline 5 & $t-\mathrm{Bu}$ & $2-A C$ & $3 e$ & 1.5 & $16 \mathrm{~h}$ & 99 \\
\hline 6 & $t-\mathrm{Bu}$ & $2-A C$ & $3 e$ & 1.3 & $30 \mathrm{~min}$ & 99 \\
\hline $7^{b}$ & Me & 4-OMe & $4 a$ & 1.3 & $96 \mathrm{~h}$ & 68 \\
\hline
\end{tabular}

a Standard reaction conditions: 1 (0.115 mmol, 1 equiv.), 2 (0.150 mmol, 1.3 equiv.), $\mathrm{MeOH}(1 \mathrm{~mL})$, rt, under air, approx. $30 \mathrm{~min}$; isolated yield.

${ }^{b}$ Reaction performed in $\mathrm{H}_{2} \mathrm{O}$, conversion determined by ${ }^{1} \mathrm{H}$ NMR analysis.

$\mathbf{2}$ and prolonged reaction time were required in this case. Also, the coupling using mesityldiazonium salt was more sluggish; additional portion of the diazonium salt and longer reaction time $(2 \mathrm{~h})$ were needed to reach full conversion but, rewardingly, under such a modified protocol 3d was afforded in high $87 \%$ yield. The reaction occurs with a comparable outcome when using the less hindered 2-(methyl)$1 \mathrm{H}$-indole (1b), furnishing the coupling products $\mathbf{4 a - c}$ in very high yields. The mesityl-derived azoindole $4 \mathbf{d}$ was obtained in $85 \%$ yield using 2 equivalents of the diazo salt partner. Functionalized indole substrates bearing $\mathrm{F}, \mathrm{Cl}$, and Me motifs could also be converted into the expected products $\mathbf{4 e -} \mathbf{e}$ in excellent yields. Of note is that this reaction is not specific to $1 \mathrm{H}$-indoles, and diazo-( $N$-methyl $)$ indoles $\mathbf{5 a - c}$ were also synthesized successfully. In contrast, acyl- 

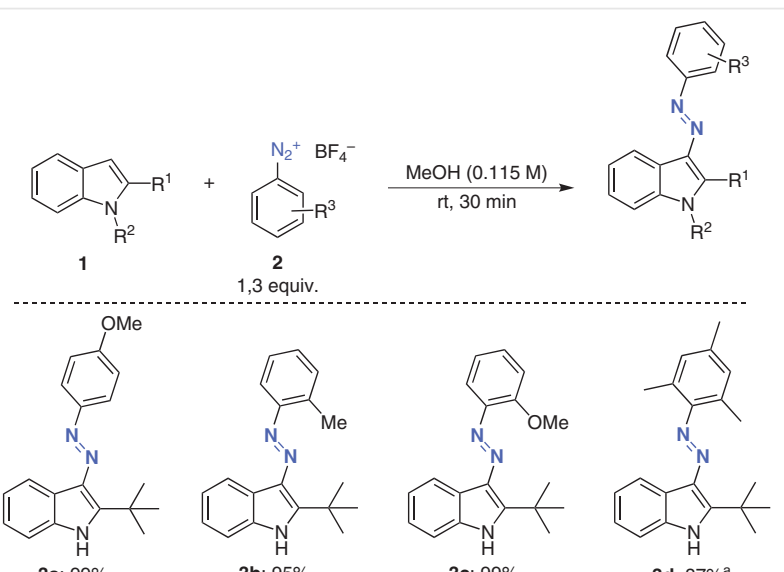

3a: $99 \%$

3b: $95 \%$

3c: $99 \%$
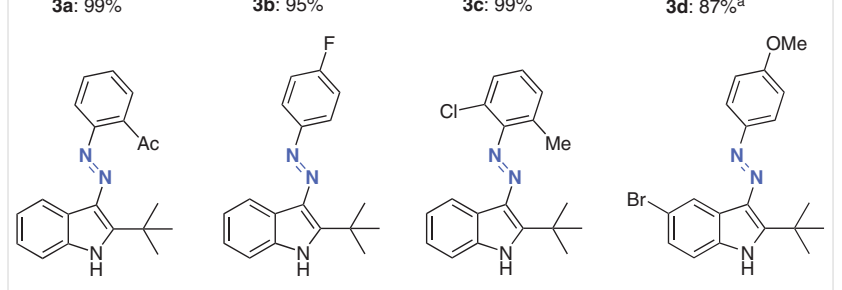

3g: $93 \%$
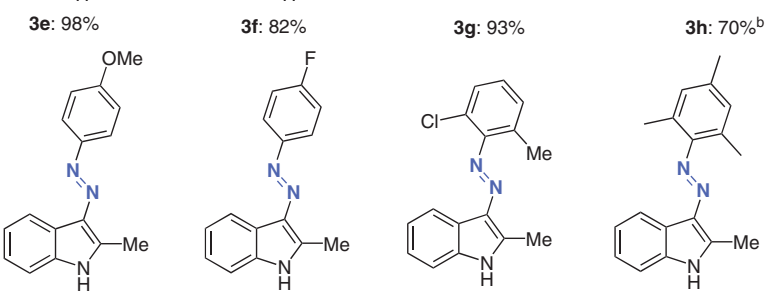

4b: $99 \%$

4a: $98 \%$
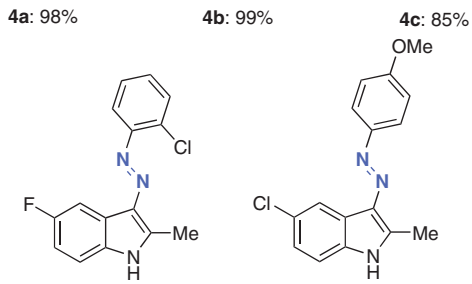

4f: $84 \%^{c}$

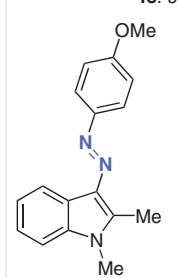

$\mathrm{Me}$
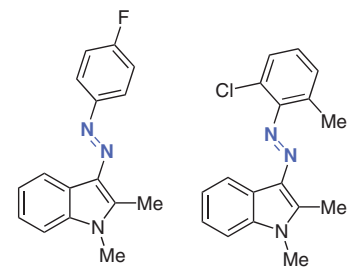

5a: $90 \%$

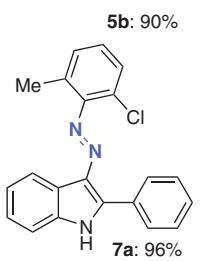

5c: $47 \%$

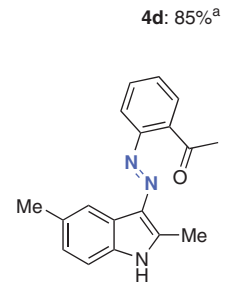

4g: $99 \%$
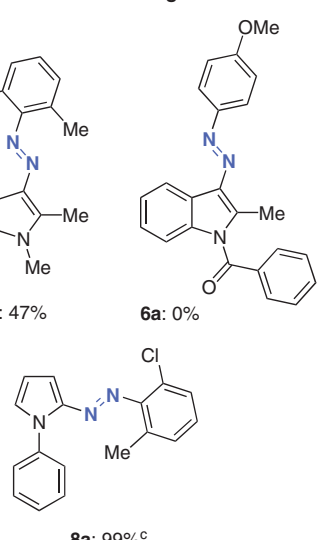

Scheme 1 Scope of C3-diazenylation of indoles. Isolated yields are shown. a) an additional portion of 0.7 equiv. of 2 was added after 30 min, and stirred for $2 \mathrm{~h}$; b) an additional portion of 0.7 equiv. of 2 was added twice after $30 \mathrm{~min}$, and stirred for $1 \mathrm{~h}$; c) an additional portion of 0.7 equiv. of 2 was added after $30 \mathrm{~min}$, and stirred for $30 \mathrm{~min}$. protected indole turned out to be ineffective. Finally, under the standard reaction conditions, 2-phenylindole was converted into the corresponding diazo compound $\mathbf{7 a}$ in $96 \%$ yield and pyrrole-derived substrate undergoes selective C2functionalization delivering 8a in quantitative yield.

Importantly, the reaction is also efficient even at 50 times larger scale ( $5.75 \mathrm{mmol}, 754 \mathrm{mg}$ of $\mathbf{1 b}$ ) and appealing product $4 \mathbf{a}$ could hence be isolated in quantitative yield (Scheme 2). However, larger excess of $\mathbf{2 a}$ (2 equiv.) and slightly longer reaction time $(1 \mathrm{~h})$ were critical to reach full conversion.

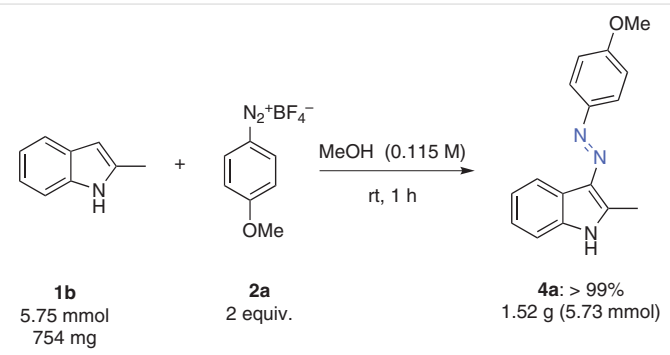

Scheme 2 Large-scale synthesis

Subsequently, in order to gather additional information about the newly synthesized compounds, their UV/Vis absorption spectra were recorded (Figure 2). Interestingly, all compounds present rather similar absorption patterns, ranging from $353 \mathrm{~nm}$ (for 3-azaindoles bearing mesityl
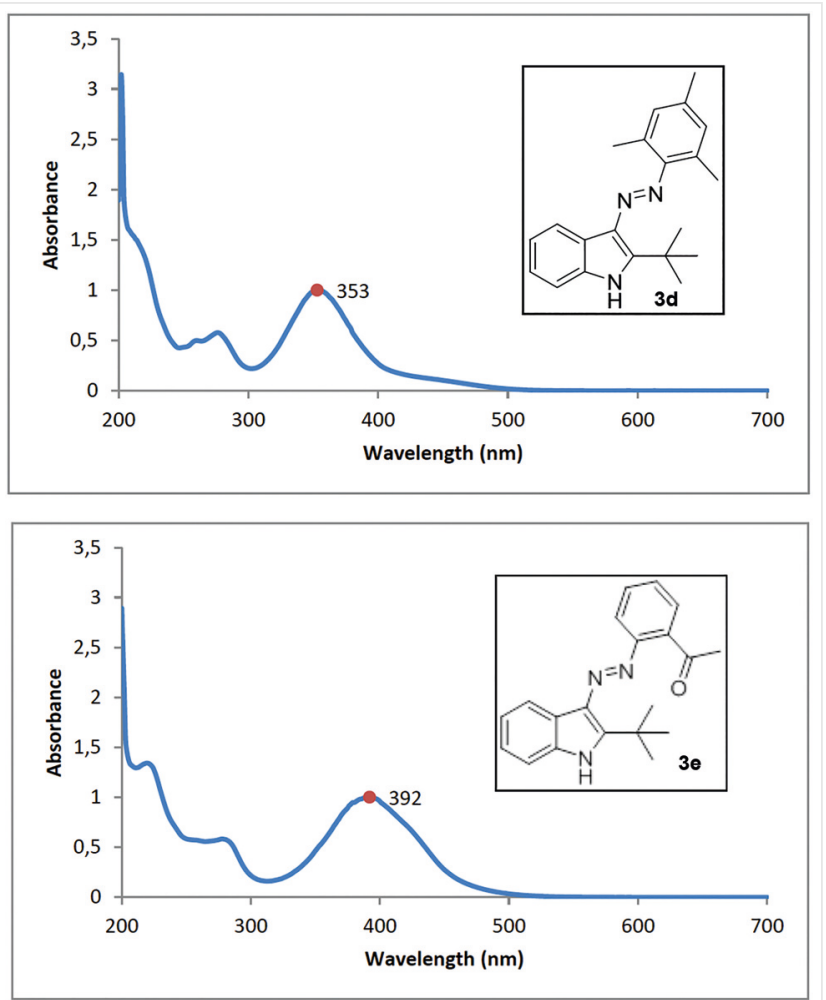

Figure 2 Examples of UV/Vis absorption spectra of the selected products 
motif such as $\mathbf{3 d}$ and $\mathbf{4 d}$ ) to $393 \mathrm{~nm}$ (for 3-azoindole featuring 2-Ac phenyl motif such as $\mathbf{3 e}$ ). Besides, the initial testes of cis-trans photoisomerization indicate that this process is relatively fast, inferior to the minute scale.

In conclusion, we have described herein a very efficient synthesis of original, highly substituted 3 -azoindoles. The coupling occurs via metal-free $\mathrm{C}-\mathrm{H}$ diazenylation of indoles, using aryldiazonium salts as coupling partners. Remarkably, the reaction does not require addition of a catalyst and performs smoothly at room temperature within few minutes delivering the expected products in quantitative yields in most of the cases. This sustainable, particularly mild and atom-economical protocol is highly tolerant towards various functionalities, furnishing a library of interesting scaffolds. These unprecedented molecules appear as privileged candidates for original photoswitch design. Besides, the simplicity of this protocol renders it perfectly suitable to be used in late-modification of sophisticated indole-containing drugs.

All the reactions were performed under air atmosphere, using tube reactors $(10 \mathrm{~mL})$. Chemicals and solvents (suppliers: Aldrich, Alfa Aesar, Fluorochem, TCI) were directly used without further purification. Technical grade solvents for purification were used without further purification or distillation. ${ }^{1} \mathrm{H},{ }^{13} \mathrm{C}$, and ${ }^{19} \mathrm{~F}$ NMR spectra were recorded in $\mathrm{CDCl}_{3}$ or acetone- $d_{6}$ at $\mathrm{rt}$ on Bruker, Avance $400(400 \mathrm{MHz})$ or Avance III-HD (500 MHz) spectrometers and FID was processed in MestreNova software. Chemical shits were referenced to residual solvent peaks and reported in ppm (i.e., $\mathrm{CDCl}_{3}$ referenced at 7.26 and $77.16 \mathrm{ppm}$ respectively and acetone- $d_{6}$ referenced at $2.05 \mathrm{ppm}$ ). Standard abbreviations were used for NMR spectra to represent the signal multiplicity. The coupling constants were reported in hertz $(\mathrm{Hz})$. Thin-layer chromatography (TLC) were carried out on precoated aluminum sheets (Merck $60-\mathrm{F}_{254}$ plates) and the components were visualized by observation under UV light at $254 \mathrm{~nm}$. Products were purified by column chromatography on $40-63$ mesh silica gel, $\mathrm{SiO}_{2}$. HRMS measurements were carried out by Service de Spectrométrie de Masse de l'Institut de Chimie at the University of Strasbourg.

The preparation of starting aryldiazonium tetrafluoroborates $\mathbf{2}$ and indoles $\mathbf{1}$ are provided in the Supporting Information.

\section{3-Azoindoles; General Procedure}

A $10 \mathrm{~mL}$ reaction tube equipped with magnetic stir bar was filled with indole derivative 1 ( $0.115 \mathrm{mmol}, 1$ equiv. $)$ and diazonium tetrafluoroborate salt 2 ( $0.150 \mathrm{mmol}, 1.3$ equiv.) under air. Then, anhydrous $\mathrm{MeOH}(1 \mathrm{~mL})$ was added, the reaction mixture turned immediately to a deep dark red color. The resulting mixture was stirred at rt for $30 \mathrm{~min}$. Afterwards, the reaction mixture was filtered through a short pad of silica gel. The reaction tube and the pad of silica gel were washed with DCM until the disappearance of color of the filtrate $(\sim 100 \mathrm{~mL})$. The solvent was removed under reduced pressure and the resulting highly colored solid was dried under vacuum to give the expected pure product.

(E)-2-(tert-Butyl)-3-[(4-methoxyphenyl)diazenyl]-1H-indole (3a) Deep orange solid; yield: $35 \mathrm{mg}(99 \%, 0.114 \mathrm{mmol})$.
${ }^{1} \mathrm{H}$ NMR $\left(\mathrm{CDCl}_{3}, 400 \mathrm{MHz}\right): \delta=8.61-8.54(\mathrm{~m}, 1 \mathrm{H}), 8.28(\mathrm{br} \mathrm{s}, 1 \mathrm{H})$, 7.86 (d, $J=8.9 \mathrm{~Hz}, 2 \mathrm{H}$ ), 7.37-7.30 (m, $1 \mathrm{H}), 7.30-7.18$ (m, $2 \mathrm{H}), 7.01$ $(\mathrm{d}, J=9.0 \mathrm{~Hz}, 2 \mathrm{H}), 3.89(\mathrm{~s}, 3 \mathrm{H}), 1.69(\mathrm{~s}, 9 \mathrm{H})$.

${ }^{13} \mathrm{C}$ NMR $\left(\mathrm{CDCl}_{3}, 101 \mathrm{MHz}\right): \delta=160.21,151.70,148.76,133.68$, 123.33, 123.32, 123.31, 123.25, 122.96, 120.49, 114.20, 110.69, 55.67, $34.11,31.01$.

HRMS (ESI): $m / z[M+H]^{+}$calcd for $\mathrm{C}_{19} \mathrm{H}_{22} \mathrm{~N}_{3} \mathrm{O}$ : 308.1757; found: 308.1754.

(E)-2-(tert-Butyl)-3-(o-tolyldiazenyl)-1H-indole (3b)

Deep orange solid; yield: $32 \mathrm{mg}$ (95\%, $0.110 \mathrm{mmol}$ ).

${ }^{1} \mathrm{H} \mathrm{NMR}\left(\mathrm{CDCl}_{3}, 400 \mathrm{MHz}\right): \delta=8.51(\mathrm{dd}, J=7.4,1.8 \mathrm{~Hz}, 1 \mathrm{H}), 8.33$ (br s, $1 \mathrm{H}), 7.70-7.63(\mathrm{~m}, 1 \mathrm{H}), 7.38-7.32(\mathrm{~m}, 2 \mathrm{H}), 7.31-7.26$ ( $\mathrm{m}, 3 \mathrm{H}), 7.26-$ $7.22(\mathrm{~m}, 1 \mathrm{H}), 2.81(\mathrm{~s}, 3 \mathrm{H}), 1.71(\mathrm{~s}, 9 \mathrm{H})$.

${ }^{13} \mathrm{C}$ NMR $\left(\mathrm{CDCl}_{3}, 101 \mathrm{MHz}\right): \delta=152.81,152.34,136.60,133.71$, $132.50,131.13,128.57,126.41,123.50,123.33,123.13,120.25$, 114.96, 110.78, 34.21, 31.05, 18.49 .

HRMS (ESI): $m / z[M+H]^{+}$calcd for $\mathrm{C}_{19} \mathrm{H}_{22} \mathrm{~N}_{3}$ : 292.1808; found: 292.1801.

(E)-2-(tert-Butyl)-3-[(2-methoxyphenyl)diazenyl]-1H-indole (3c) Deep red solid; yield: $35 \mathrm{mg}$ (99\%, $0.114 \mathrm{mmol})$.

${ }^{1} \mathrm{H} \mathrm{NMR}\left(\mathrm{CDCl}_{3}, 400 \mathrm{MHz}, 333 \mathrm{~K}\right): \delta=8.59$ (br s, $\left.1 \mathrm{H}\right), 8.50$ (d, $J=7.4$ $\mathrm{Hz}, 1 \mathrm{H}), 7.68$ (dd, $J=7.9,1.7 \mathrm{~Hz}, 1 \mathrm{H}), 7.38(\mathrm{~d}, J=7.6 \mathrm{~Hz}, 1 \mathrm{H}), 7.29-$ $7.23(\mathrm{~m}, 3 \mathrm{H}$ ), 7.08 (dd, $J=8.2,1.2 \mathrm{~Hz}, 1 \mathrm{H}), 7.03$ (td, $J=7.6,1.2 \mathrm{~Hz}, 1$ $\mathrm{H}), 4.07$ (s, $3 \mathrm{H}), 1.69$ (s, $9 \mathrm{H})$.

${ }^{13} \mathrm{C} \mathrm{NMR}\left(\mathrm{CDCl}_{3}, 101 \mathrm{MHz}, 333 \mathrm{~K}\right): \delta=155.66,153.34,143.87,133.79$, $132.55,129.10,124.36,123.81,123.34,121.30,120.79,116.30$, 113.38, 111.85, 56.99, 34.56, 31.01.

HRMS (ESI): $m / z[M+H]^{+}$calcd for $\mathrm{C}_{19} \mathrm{H}_{22} \mathrm{~N}_{3} \mathrm{O}$ : 308.1757; found: 308.1748 .

\section{(E)-2-(tert-Butyl)-3-(mesityldiazenyl)-1H-indole (3d)}

Prepared according to the general procedure, with a following modification: an additional portion of 0.7 equiv of mesityldiazonium tetrafluoroborate was added after $30 \mathrm{~min}$; stirred for $2 \mathrm{~h}$; deep orange solid; yield: $32 \mathrm{mg}$ ( $87 \%, 0.100 \mathrm{mmol})$.

${ }^{1} \mathrm{H} \mathrm{NMR}\left(\mathrm{CDCl}_{3}, 400 \mathrm{MHz}\right): \delta=8.57-8.48(\mathrm{~m}, 1 \mathrm{H}), 8.29(\mathrm{br} \mathrm{s}, 1 \mathrm{H})$, 7.40-7.33 (m, 1 H), 7.31-7.21 (m, 2 H), 6.96 (s, 2 H), 2.42 (s, 6 H), 2.35 (s, $3 \mathrm{H}), 1.65(\mathrm{~s}, 9 \mathrm{H})$.

${ }^{13} \mathrm{C}$ NMR $\left(\mathrm{CDCl}_{3}, 101 \mathrm{MHz}\right): \delta=152.00,150.67,136.14,133.45$, 132.31, 130.35, 129.69, 123.35, 123.25, 123.19, 120.32, 110.63, 34.07, $30.90,21.11,19.60$.

HRMS (ESI): $m / z[M+H]^{+}$calcd for $\mathrm{C}_{21} \mathrm{H}_{26} \mathrm{~N}_{3}$ : 320.2121; found: 320.2122 .

\section{(E)-1-(2-\{[2-(tert-Butyl)-1H-indol-3-yl]diazenyl\}phenyl)ethan-1-} one (3e)

Deep orange solid; yield: $36 \mathrm{mg}(98 \%, 0.113 \mathrm{mmol})$.

${ }^{1} \mathrm{H} \mathrm{NMR}\left(\mathrm{CDCl}_{3}, 400 \mathrm{MHz}\right): \delta=12.87(\mathrm{br} \mathrm{s}, 1 \mathrm{H}), 8.35(\mathrm{~d}, J=6.9 \mathrm{~Hz}, 1$ H), $7.97(\mathrm{~d}, J=8.5 \mathrm{~Hz}, 1 \mathrm{H}), 7.93(\mathrm{~d}, J=7.9 \mathrm{~Hz}, 1 \mathrm{H}), 7.64(\mathrm{t}, J=7.8 \mathrm{~Hz}, 1$ H), $7.59(\mathrm{~d}, J=6.5 \mathrm{~Hz}, 1 \mathrm{H}), 7.45-7.38(\mathrm{~m}, 2 \mathrm{H}), 7.11(\mathrm{t}, J=7.5 \mathrm{~Hz}, 1 \mathrm{H})$, $2.75(\mathrm{~s}, 3 \mathrm{H}), 1.60(\mathrm{~s}, 9 \mathrm{H})$.

${ }^{13} \mathrm{C}$ NMR $\left(\mathrm{CDCl}_{3}, 101 \mathrm{MHz}\right): \delta=202.25,179.13,145.95,141.96$, $135.64,132.13,130.49,126.41,122.52,121.72,121.47,120.90$, $119.83,114.96,36.53,30.53,28.27$ (1 C undetected due to overlapping). 
HRMS (ESI): $m / z[M+H]^{+}$calcd for $\mathrm{C}_{20} \mathrm{H}_{22} \mathrm{~N}_{3} \mathrm{O}$ : 320.1757; found: 320.1744 .

(E)-2-(tert-Butyl)-3-[(4-fluorophenyl)diazenyl]-1H-indole (3f) Deep yellow solid; yield: $28 \mathrm{mg}(82 \%, 0.095 \mathrm{mmol})$.

${ }^{1} \mathrm{H} \mathrm{NMR}\left(\mathrm{CDCl}_{3}, 400 \mathrm{MHz}\right): \delta=8.59-8.52(\mathrm{~m}, 1 \mathrm{H}), 8.34(\mathrm{br} \mathrm{s}, 1 \mathrm{H})$, 7.88-7.83 (m, 2 H), 7.38-7.33 (m, 1 H), 7.31-7.22 (m, 2 H), 7.20-7.12 (m, $2 \mathrm{H}), 1.69$ (s, $9 \mathrm{H})$.

${ }^{13} \mathrm{C}$ NMR $\left(\mathrm{CDCl}_{3}, 126 \mathrm{MHz}\right): \delta=162.97(\mathrm{~d}, J=248.0 \mathrm{~Hz}), 152.87$, $150.89(\mathrm{~d}, J=3.0 \mathrm{~Hz}), 133.70,131.57,123.59,123.46(\mathrm{~d}, J=8.5 \mathrm{~Hz})$, 123.27, 123.23, 120.33, 115.82 (d, J = 22.5 Hz), 110.80, 34.19, 31.03 . ${ }^{19} \mathrm{~F} \mathrm{NMR}\left(\mathrm{CDCl}_{3}, 376 \mathrm{MHz}\right): \delta=-113.73(\mathrm{~s}, 1 \mathrm{~F})$.

HRMS (ESI): $m / z[M+\mathrm{H}]^{+}$calcd for $\mathrm{C}_{18} \mathrm{H}_{19} \mathrm{FN}_{3}$ : 296.1557; found: 296.1544.

\section{(E)-2-(tert-Butyl)-3-[(2-chloro-6-methylphenyl)diazenyl]-1H- indole (3g)}

Deep orange solid; yield: $35 \mathrm{mg}$ (93\%, $0.107 \mathrm{mmol}$ ).

${ }^{1} \mathrm{H} \mathrm{NMR}\left(\mathrm{CDCl}_{3}, 500 \mathrm{MHz}\right): \delta=8.56(\mathrm{dd}, J=6.5,2.1 \mathrm{~Hz}, 1 \mathrm{H}), 8.39$ (br s, $1 \mathrm{H}), 7.38-7.34$ (m, $2 \mathrm{H}), 7.30-7.24(\mathrm{~m}, 2 \mathrm{H}), 7.17$ (ddd, $J=7.6,1.5,0.7$ $\mathrm{Hz}, 1 \mathrm{H}), 7.09$ (t, J = 7.8 Hz, $1 \mathrm{H}), 2.39(\mathrm{~s}, 3 \mathrm{H}), 1.65(\mathrm{~s}, 9 \mathrm{H})$.

${ }^{13} \mathrm{C} \mathrm{NMR}\left(\mathrm{CDCl}_{3}, 126 \mathrm{MHz}\right): \delta=153.84,150.84,133.59,132.55$, $131.56,129.71,128.11,127.33,126.78,123.75,123.72,123.40$, 120.17, 110.73, 34.26, 30.92, 19.47.

HRMS (ESI): $m / z[\mathrm{M}+\mathrm{H}]^{+}$calcd for $\mathrm{C}_{19} \mathrm{H}_{21} \mathrm{ClN}_{3}$ : 326.1419; found: 326.1408 .

\section{(E)-5-Bromo-2-(tert-butyl)-3-[(4-methoxyphenyl)diazenyl]-1H- indole (3h)}

Prepared according to the general procedure, with a following modification: two additional portions of 0.7 equiv of mesityldiazonium tetrafluoroborate were added after $30 \mathrm{~min}$ and $1 \mathrm{~h}$; stirred for $1 \mathrm{~h}$; deep orange solid; yield: $31 \mathrm{mg}(70 \%, 0.0803 \mathrm{mmol})$.

${ }^{1} \mathrm{H} \mathrm{NMR}\left(\mathrm{CDCl}_{3}, 500 \mathrm{MHz}\right): \delta=8.72(\mathrm{~d}, J=1.9 \mathrm{~Hz}, 1 \mathrm{H}), 8.30(\mathrm{br} \mathrm{s}, 1 \mathrm{H})$, $7.86(\mathrm{~d}, J=9.0 \mathrm{~Hz}, 2 \mathrm{H}), 7.31(\mathrm{dd}, J=8.5,2.0 \mathrm{~Hz}, 1 \mathrm{H}), 7.20(\mathrm{~d}, J=$ $8.5 \mathrm{~Hz}, 1 \mathrm{H}), 7.01(\mathrm{~d}, J=8.9 \mathrm{~Hz}, 2 \mathrm{H}), 3.89(\mathrm{~s}, 3 \mathrm{H}), 1.67(\mathrm{~s}, 9 \mathrm{H})$.

${ }^{13} \mathrm{C}$ NMR $\left(\mathrm{CDCl}_{3}, 126 \mathrm{MHz}\right): \delta=160.48,152.42,148.49,132.29$, 130.63, 126.07, 125.73, 123.49, 121.91, 116.10, 114.23, 112.13, 55.68, $34.15,30.89$.

HRMS (ESI): $m / z[\mathrm{M}+\mathrm{H}]^{+}$calcd for $\mathrm{C}_{19} \mathrm{H}_{21} \mathrm{BrN}_{3} \mathrm{O}$ : 386.0863; found: 386.0847.

\section{(E)-3-[(4-Methoxyphenyl)diazenyl]-2-methyl-1H-indole (4a)}

Deep red solid; yield: $30 \mathrm{mg}$ (98\%, $0.113 \mathrm{mmol})$.

${ }^{1} \mathrm{H}$ NMR $\left(\mathrm{CDCl}_{3}, 400 \mathrm{MHz}\right): \delta=8.57-8.50(\mathrm{~m}, 1 \mathrm{H}), 8.18$ (br s, $\left.1 \mathrm{H}\right)$, $7.89(\mathrm{~d}, J=8.9 \mathrm{~Hz}, 2 \mathrm{H}), 7.30-7.26(\mathrm{~m}, 1 \mathrm{H}), 7.26-7.20(\mathrm{~m}, 2 \mathrm{H}), 7.01$ (d, $J=9.0 \mathrm{~Hz}, 2 \mathrm{H}), 3.88(\mathrm{~s}, 3 \mathrm{H}), 2.80(\mathrm{~s}, 3 \mathrm{H})$.

${ }^{13} \mathrm{C}$ NMR $\left(\mathrm{CDCl}_{3}, 126 \mathrm{MHz}\right): \delta=160.33,148.61,141.70,135.10$, 132.66, 123.45, 123.26, 122.71, 122.57, 119.88, 114.18, 110.55, 55.66, 11.67.

HRMS (ESI): $m / z[\mathrm{M}+\mathrm{H}]^{+}$calcd for $\mathrm{C}_{16} \mathrm{H}_{16} \mathrm{~N}_{3} \mathrm{O}$ : 266.1285; found: 266.1288 .

\section{(E)-3-[(4-Fluorophenyl)diazenyl]-2-methyl-1H-indole (4b)}

Deep yellow solid; yield: $29 \mathrm{mg}$ (99\%, $0.115 \mathrm{mmol})$.
${ }^{1} \mathrm{H} \mathrm{NMR}\left(\mathrm{CDCl}_{3}, 400 \mathrm{MHz}\right): \delta=8.54-8.48(\mathrm{~m}, 1 \mathrm{H}), 8.28(\mathrm{br} \mathrm{s}, 1 \mathrm{H})$, 7.91-7.86 (m, 2 H), 7.33-7.24 (m, 3 H), 7.20-7.12 (m, 2 H), 2.82 (s, $3 \mathrm{H})$.

${ }^{13} \mathrm{C}$ NMR $\left(\mathrm{CDCl}_{3}, 126 \mathrm{MHz}\right): \delta=163.08(\mathrm{~d}, J=248.2 \mathrm{~Hz}), 150.75(\mathrm{~d}, J=$ $1.8 \mathrm{~Hz}$ ), 142.92, 135.14, 132.81, 123.75, 123.41 (d, $J=8.4 \mathrm{~Hz}), 123.03$, $122.57,119.70,115.80(\mathrm{~d}, J=22.7 \mathrm{~Hz}), 110.65,11.72$.

${ }^{19} \mathrm{~F} \mathrm{NMR}\left(\mathrm{CDCl}_{3}, 376 \mathrm{MHz}\right): \delta=-113.55(\mathrm{~s}, 1 \mathrm{~F})$.

HRMS (ESI): $m / z[M+H]^{+}$calcd for $\mathrm{C}_{15} \mathrm{H}_{13} \mathrm{FN}_{3}$ : 254.1088; found: 254.1076.

\section{(E)-3-[(2-Chloro-6-methylphenyl)diazenyl]-2-methyl-1H-indole}

(4c)

Deep orange solid; yield: $28 \mathrm{mg}$ (85\%, $0.099 \mathrm{mmol})$.

${ }^{1} \mathrm{H} \mathrm{NMR}\left(\mathrm{CDCl}_{3}, 400 \mathrm{MHz}\right): \delta=8.50(\mathrm{~d}, J=7.4 \mathrm{~Hz}, 1 \mathrm{H}), 8.31(\mathrm{br} \mathrm{s}, 1 \mathrm{H})$, $7.35(\mathrm{dd}, J=8.0,0.7 \mathrm{~Hz}, 1 \mathrm{H}), 7.33-7.24(\mathrm{~m}, 3 \mathrm{H}), 7.16(\mathrm{~d}, J=7.6 \mathrm{~Hz}, 1$ H), 7.09 (t, J = 7.7 Hz, $1 \mathrm{H}), 2.79$ (s, $3 \mathrm{H}), 2.42(\mathrm{~s}, 3 \mathrm{H})$.

${ }^{13} \mathrm{C}$ NMR $\left(\mathrm{CDCl}_{3}, 126 \mathrm{MHz}\right): \delta=150.46,143.72,135.11,133.74$, $131.57,130.05,128.20,127.05,123.90,123.89,123.44,122.62$, 119.42, 110.61, 19.61, 11.64.

HRMS (ESI): $m / z[\mathrm{M}+\mathrm{H}]^{+}$calcd for $\mathrm{C}_{16} \mathrm{H}_{15} \mathrm{ClN}_{3}$ : 284.0949; found: 284.0946 .

\section{(E)-3-(Mesityldiazenyl)-2-methyl-1H-indole (4d)}

Prepared according to the general procedure, with a following modification: an additional portion of 0.7 equiv of mesityldiazonium tetrafluoroborate was added after $30 \mathrm{~min}$; stirred for $2 \mathrm{~h}$.

Deep yellow solid; yield: $27 \mathrm{mg}$ (85\%, $0.097 \mathrm{mmol})$.

${ }^{1} \mathrm{H} \mathrm{NMR}\left(\mathrm{CDCl}_{3}, 400 \mathrm{MHz}\right): \delta=8.47-8.41(\mathrm{~m}, 1 \mathrm{H}), 8.20$ (br s, $\left.1 \mathrm{H}\right)$, 7.33-7.23 (m, 3 H), 6.95 (s, 2 H), 2.77 (s, 3 H), 2.45 (s, 6 H), 2.33 (s, 3 $\mathrm{H})$.

${ }^{13} \mathrm{C}$ NMR $\left(\mathrm{CDCl}_{3}, 126 \mathrm{MHz}\right): \delta=150.24,142.10,136.47,135.06$, 133.41, 130.79, 129.94, 123.47, 122.92, 122.37, 119.50, 110.56, 21.15, $19.68,11.58$.

HRMS (ESI): $m / z[\mathrm{M}+\mathrm{H}]^{+}$calcd for $\mathrm{C}_{18} \mathrm{H}_{20} \mathrm{~N}_{3}$ : 278.1652; found: 278.1648 .

\section{(E)-3-[(2-Chlorophenyl)diazenyl]-5-fluoro-2-methyl-1H-indole}

(4e)

Deep yellow solid; yield: $32 \mathrm{mg}$ (97\%, $0.111 \mathrm{mmol})$.

${ }^{1} \mathrm{H} \mathrm{NMR}\left(\mathrm{CDCl}_{3}, 500 \mathrm{MHz}\right): \delta=8.32(\mathrm{dd}, J=9.7,2.7 \mathrm{~Hz}, 1 \mathrm{H}), 8.29$ (br s, $1 \mathrm{H}), 7.80$ (dd, $J=7.9,1.8 \mathrm{~Hz}, 1 \mathrm{H}$ ), 7.55 (dd, $J=7.8,1.5 \mathrm{~Hz}, 1 \mathrm{H}$ ), 7.34$7.30(\mathrm{~m}, 1 \mathrm{H}), 7.28(\mathrm{dd}, J=7.7,1.8 \mathrm{~Hz}, 1 \mathrm{H}), 7.19(\mathrm{dd}, J=8.7,4.3 \mathrm{~Hz}, 1$ $\mathrm{H}), 6.98(\mathrm{td}, J=8.9,2.6 \mathrm{~Hz}, 1 \mathrm{H}), 2.82(\mathrm{~s}, 3 \mathrm{H})$.

${ }^{13} \mathrm{C}$ NMR $\left(\mathrm{CDCl}_{3}, 126 \mathrm{MHz}\right): \delta=160.30(\mathrm{~d}, J=237.9 \mathrm{~Hz}), 149.83$, $145.08,134.19$ (d, $J=3.9 \mathrm{~Hz}), 134.10,131.48,130.56,129.58,127.19$, $120.02(\mathrm{~d}, J=11.3 \mathrm{~Hz}), 116.95,111.81(\mathrm{~d}, J=26.3 \mathrm{~Hz}), 111.23(\mathrm{~d}, J=$ $9.5 \mathrm{~Hz}), 108.63(\mathrm{~d}, J=25.6 \mathrm{~Hz}), 11.81$.

${ }^{19} \mathrm{~F} \mathrm{NMR}\left(\mathrm{CDCl}_{3}, 376 \mathrm{MHz}\right): \delta=-120.04(\mathrm{~s}, 1 \mathrm{~F})$

HRMS (ESI): $m / z[\mathrm{M}+\mathrm{H}]^{+}$calcd for $\mathrm{C}_{15} \mathrm{H}_{12} \mathrm{ClFN}_{3}$ : 288.0698; found: 288.0686

\section{(E)-5-Chloro-3-[(4-methoxyphenyl)diazenyl]-2-methyl-1H-indole} (4f)

Prepared according to the general procedure, but by using another 0.7 equiv of the corresponding aryldiazonium tetrafluoroborate after 30 min; stirred for $30 \mathrm{~min}$.

Deep orange solid; yield: $29 \mathrm{mg}(84 \%, 0.097 \mathrm{mmol})$. 
${ }^{1} \mathrm{H} \mathrm{NMR}\left(\mathrm{CDCl}_{3}, 400 \mathrm{MHz}\right): \delta=8.51(\mathrm{dd}, J=1.9,0.8 \mathrm{~Hz}, 1 \mathrm{H}), 8.26(\mathrm{br} \mathrm{s}$, $1 \mathrm{H}), 7.88(\mathrm{~d}, J=9.0 \mathrm{~Hz}, 2 \mathrm{H}), 7.19(\mathrm{~d}, J=0.8 \mathrm{~Hz}, 1 \mathrm{H}), 7.18(\mathrm{~d}, J=1.9 \mathrm{~Hz}$, $1 \mathrm{H}), 7.00(\mathrm{~d}, J=9.0 \mathrm{~Hz}, 2 \mathrm{H}), 3.89(\mathrm{~s}, 3 \mathrm{H}), 2.80(\mathrm{~s}, 3 \mathrm{H})$.

${ }^{13} \mathrm{C}$ NMR $\left(\mathrm{CDCl}_{3}, 101 \mathrm{MHz}\right): \delta=160.60,148.22,142.74,133.42$, 132.03, 128.28, 123.62, 123.38, 122.14, 120.74, 114.23, 111.54, 55.68, 11.73.

HRMS (ESI): $m / z[\mathrm{M}+\mathrm{H}]^{+}$calcd for $\mathrm{C}_{16} \mathrm{H}_{15} \mathrm{ClN}_{3} \mathrm{O}$ : 300.0898; found: 300.0888 .

\section{(E)-1-\{2-[(2,5-Dimethyl-1H-indol-3-yl)diazenyl]phenyl\}ethan-1-} one (4g)

Deep orange solid; yield: $33 \mathrm{mg}$ (quant, $0.115 \mathrm{mmol}$ ).

${ }^{1} \mathrm{H} \mathrm{NMR}\left(\mathrm{CDCl}_{3}, 500 \mathrm{MHz}\right): \delta=8.15(\mathrm{~s}, 1 \mathrm{H}), 8.03(\mathrm{~d}, J=8.3 \mathrm{~Hz}, 1 \mathrm{H})$, $7.88(\mathrm{~d}, J=7.8 \mathrm{~Hz}, 1 \mathrm{H}), 7.63$ (ddd J = 8.5, 7.2, $1.5 \mathrm{~Hz}, 1 \mathrm{H}), 7.39(\mathrm{~d}, J=$ $7.9 \mathrm{~Hz}, 1 \mathrm{H}), 7.22-7.18(\mathrm{~m}, 2 \mathrm{H}), 2.72(\mathrm{~s}, 3 \mathrm{H}), 2.65(\mathrm{~s}, 3 \mathrm{H}), 2.53(\mathrm{~s}, 3 \mathrm{H})$ (NH proton not detected).

HRMS (ESI): $m / z$ [M $+\mathrm{H}]^{+}$calcd for $\mathrm{C}_{18} \mathrm{H}_{19} \mathrm{~N}_{3} \mathrm{O}$ : 292.1444; found: 292.1439.

\section{(E)-3-[(4-Methoxyphenyl)diazenyl]-1,2-dimethyl-1H-indole (5a)}

Deep orange solid; yield: $29 \mathrm{mg}$ (90\%, $0.104 \mathrm{mmol})$.

${ }^{1} \mathrm{H} \mathrm{NMR}\left(\mathrm{CDCl}_{3}, 500 \mathrm{MHz}\right): \delta=8.59-8.54(\mathrm{~m}, 1 \mathrm{H}), 7.87(\mathrm{~d}, J=9.0 \mathrm{~Hz}, 2$ H), 7.34-7.26 (m, $3 \mathrm{H}), 7.00$ (d, J = 8.9 Hz, $2 \mathrm{H}), 3.88$ (s, $3 \mathrm{H}), 3.76(\mathrm{~s}, 3$ $\mathrm{H}), 2.82(\mathrm{~s}, 3 \mathrm{H})$.

${ }^{13} \mathrm{C}$ NMR $\left(\mathrm{CDCl}_{3}, 126 \mathrm{MHz}\right): \delta=160.10,148.72,143.88,136.96,132.30$ $123.11,122.69,122.66,119.27,114.16,108.85,55.66,29.98,10.30$ (1 $C$ undetected due to overlapping).

HRMS (ESI): $m / z[M+H]^{+}$calcd for $\mathrm{C}_{17} \mathrm{H}_{18} \mathrm{~N}_{3} \mathrm{O}$ : 280.1444; found: 280.1433 .

\section{(E)-3-[(4-Fluorophenyl)diazenyl]-1,2-dimethyl-1H-indole (5b)}

Deep orange solid; yield: $33.5 \mathrm{mg}(90 \%, 0.112 \mathrm{mmol})$.

${ }^{1} \mathrm{H} \mathrm{NMR}\left(\mathrm{CDCl}_{3}, 500 \mathrm{MHz}\right): \delta=8.58-8.52(\mathrm{~m}, 1 \mathrm{H}), 7.90-7.85(\mathrm{~m}, 2 \mathrm{H})$, 7.34-7.27 (m, $3 \mathrm{H}), 7.15(\mathrm{t}, J=8.7 \mathrm{~Hz}, 2 \mathrm{H}), 3.76(\mathrm{~s}, 3 \mathrm{H}), 2.82(\mathrm{~s}, 3 \mathrm{H})$.

${ }^{13} \mathrm{C} \mathrm{NMR}\left(\mathrm{CDCl}_{3}, 126 \mathrm{MHz}\right): \delta=162.88(\mathrm{~d}, J=247.5 \mathrm{~Hz}), 150.81(\mathrm{~d}, J=$ $2.4 \mathrm{~Hz}), 145.19,137.08,132.42,123.43,123.24$ (d, $J=8.4 \mathrm{~Hz}), 123.07$, 122.66, 119.11, $115.76(\mathrm{~d}, J=22.6 \mathrm{~Hz}), 109.00,30.08,10.34$.

${ }^{19} \mathrm{~F} \mathrm{NMR}\left(\mathrm{CDCl}_{3}, 376 \mathrm{MHz}\right): \delta=-113.97(\mathrm{~s}, 1 \mathrm{~F})$.

HRMS (ESI): $m / z[M+\mathrm{H}]^{+}$calcd for $\mathrm{C}_{16} \mathrm{H}_{15} \mathrm{FN}_{3}$ : 268.1244; found: 268.1230.

\section{(E)-3-[(2-Chloro-6-methylphenyl)diazenyl]-1,2-dimethyl-1H- indole (5c)}

Deep orange solid; yield: $16 \mathrm{mg}$ (47\%, $0.054 \mathrm{mmol})$.

${ }^{1} \mathrm{H} \mathrm{NMR}\left(\mathrm{CDCl}_{3}, 500 \mathrm{MHz}\right): \delta=8.56-8.50(\mathrm{~m}, 1 \mathrm{H}), 7.37-7.27(\mathrm{~m}, 4 \mathrm{H})$, 7.16 (ddd, $J=7.6,1.5,0.8 \mathrm{~Hz}, 1 \mathrm{H}), 7.07(\mathrm{t}, J=7.7 \mathrm{~Hz}, 1 \mathrm{H}), 3.77(\mathrm{~s}, 3 \mathrm{H})$, 2.79 (s, $3 \mathrm{H}), 2.42$ (s, $3 \mathrm{H})$.

${ }^{13} \mathrm{C}$ NMR $\left(\mathrm{CDCl}_{3}, 126 \mathrm{MHz}\right): \delta=150.60,145.74,137.05,133.36$, $131.65,129.98,128.19,128.16,126.81,123.51,123.41,122.69$, 118.84, 108.93, 30.04, 19.63, 10.19 .

HRMS (ESI): $m / z[\mathrm{M}+\mathrm{H}]^{+}$calcd for $\mathrm{C}_{17} \mathrm{H}_{17} \mathrm{ClN}_{3}$ : 298.1106; found: 298.1090.

\section{(E)-3-[(2-Chloro-6-methylphenyl)diazenyl]-2-phenyl-1H-indole}

(7a)

Deep orange solid; yield: $38 \mathrm{mg}$ (96\%, $0.110 \mathrm{mmol})$.
${ }^{1} \mathrm{H} \mathrm{NMR}\left(\mathrm{CDCl}_{3}, 500 \mathrm{MHz}\right): \delta=8.67-8.63(\mathrm{~m}, 1 \mathrm{H}), 8.61(\mathrm{br} \mathrm{s}, 1 \mathrm{H})$, 8.03-7.98 (m, 2 H), 7.53-7.47 (m, 2 H), 7.46-7.42 (m, 2 H), 7.39-7.30 $(\mathrm{m}, 3 \mathrm{H}), 7.15$ (ddd, $J=7.6,1.5,0.8 \mathrm{~Hz}, 1 \mathrm{H}), 7.10(\mathrm{t}, J=7.7 \mathrm{~Hz}, 1 \mathrm{H})$, 2.39 (s, $3 \mathrm{H})$.

${ }^{13} \mathrm{C}$ NMR $\left(\mathrm{CDCl}_{3}, 126 \mathrm{MHz}\right): \delta=150.58,142.92,135.46,133.22$, $131.66,130.64,129.94,129.57,129.18,128.96,128.24,127.70$, 127.33, 124.88, 123.99, 123.87, 119.87, 111.01, 19.66.

HRMS (ESI): $m / z[\mathrm{M}+\mathrm{H}]^{+}$calcd for $\mathrm{C}_{21} \mathrm{H}_{17} \mathrm{ClN}_{3}$ : 346.1106; found: 346.1091.

\section{(E)-N-(2-Chloro-6-methylphenyl)-1-(1-phenyl-1H-pyrrol-2- yl)methanimine (8a)}

Prepared according to the general procedure, but by using another 0.7 equiv. of the corresponding aryldiazonium tetrafluoroborate after 30 $\mathrm{min}$; stirred for $30 \mathrm{~min}$; deep orange solid; yield: $34 \mathrm{mg}$ (99\%, 0.115 mmol).

${ }^{1} \mathrm{H} \mathrm{NMR}\left(\mathrm{CDCl}_{3}, 500 \mathrm{MHz}\right): \delta=7.53-7.48(\mathrm{~m}, 2 \mathrm{H}), 7.46-7.41(\mathrm{~m}, 2 \mathrm{H})$, 7.38-7.34 (m, $1 \mathrm{H}), 7.29-7.26(\mathrm{~m}, 1 \mathrm{H}), 7.23$ (dd, $J=2.8,1.6 \mathrm{~Hz}, 1 \mathrm{H})$, 7.08-7.03 (m, $2 \mathrm{H}), 6.93(\mathrm{dd}, J=4.2,1.7 \mathrm{~Hz}, 1 \mathrm{H}), 6.48(\mathrm{dd}, J=4.1,2.8$ $\mathrm{Hz}, 1 \mathrm{H}), 2.16$ (s, $3 \mathrm{H})$.

${ }^{13} \mathrm{C}$ NMR $\left(\mathrm{CDCl}_{3}, 126 \mathrm{MHz}\right): \delta=149.50,147.43,138.66,131.93$, $129.93,128.94,128.22,128.19,127.81,127.68,127.27,126.55$, $111.24,100.20,19.43$.

HRMS (ESI): $m / z[\mathrm{M}+\mathrm{H}]^{+}$calcd for $\mathrm{C}_{17} \mathrm{H}_{15} \mathrm{ClN}_{3}$ : 296.0949; found: 296.0940 .

\section{Funding Information}

This work was carried out within ANR JCJC grant '2al-Vis-Phot-CH' (ANR-15-CE29-0004-01).

\section{Acknowledgment}

L.G. acknowledges Agence Nationale de la Recherche (ANR) for the Ph.D. grant and N.J. acknowledges ANR for Master 2 fellowship. We would like to thank Dr. A. Specht and Dr. S. Lakhdar for inspiring initial photophysical study discussions.

\section{Supporting Information}

Supporting information for this article is available online at https://doi.org/10.1055/s-0039-1690048.

\section{References}

(1) For selected reviews, see: (a) Brieke, C.; Rohrbach, F.; Gottschalk, A.; Mayer, G.; Heckel, A. Angew. Chem. Int. Ed. 2012, 51, 8446. (b) Russew, M.-M.; Hecht, S. Adv. Mater. 2010, 22, 3348. (c) Pianowski, Z. L. Chem. Eur. J. 2019, 25, 5128. (d) Mutlu, H.; Geiselhart, C. M.; Barner-Kowollik, C. Mater. Horiz. 2018, 5, 162. (e) Bléger, D.; Hecht, S. Angew. Chem. Int. Ed. 2015, 54, 11338.

(2) Baroncini, M.; d'Agostino, S.; Bergamini, G.; Ceroni, P.; Comotti, A.; Sozzani, P.; Bassanetti, I.; Grepioni, F.; Hernandez, T. M.; Silvi, S.; Venturi, M.; Credi, A. Nat. Chem. 2015, 7, 634.

(3) Hirshberg, Y. J. Am. Chem. Soc. 1956, 78, 2304. 
(4) Roke, D.; Stuckhardt, C.; Danowski, W.; Wezenberg, S. J.; Feringa, B. L. Angew. Chem. Int. Ed. 2018, 57, 10515.

(5) (a) Barber, R. W.; McFadden, M. E.; Hu, X.; Robb, M. J. Synlett 2019, 30, 1725. (b) Ihrig, S. P.; Eisenreich, F.; Hecht, S. Chem. Commun. 2019, 55, 4290.

(6) (a) Beauté, L.; McClenaghan, N.; Lecommandoux, S. Adv. Drug Deliv. Rev. 2019, 138, 148. (b) Jia, S.; Fong, W.-K.; Graham, B.; Boyd, B. J. Chem. Mater. 2018, 30, 2873.

(7) Borowiak, M.; Nahaboo, W.; Reynders, M.; Nekolla, K.; Jalinot, P.; Hasserodt, J.; Rehberg, M.; Delattre, M.; Zahler, S.; Vollmar, A.; Trauner, D.; Thorn-Seshold, O. Cell 2015, 162, 403.

(8) Andréasson, J.; Pischel, U.; Straight, S. D.; Moore, T. A.; Moore, A. L.; Gust, D. J. Am. Chem. Soc. 2011, 133, 11641.

(9) (a) Goldau, T.; Murayama, K.; Brieke, C.; Asanuma, H.; Heckel, A. Chem. Eur. J. 2015, 21, 17870. (b) Nakasone, Y.; Ooi, H.; Kamiya, Y.; Asanuma, H.; Terazima, M. J. Am. Chem. Soc. 2016, 138, 9001. (c) Rullo, A.; Reiner, A.; Reiter, A.; Trauner, D.; Isacoff, E. Y.; Woolley, G. A. Chem. Commun. 2014, 50, 14613. (d) Goldau, T.; Murayama, K.; Brieke, C.; Steinwand, S.; Mondal, P.; Biswas, M.; Burghardt, I.; Wachtveitl, J.; Asanuma, H.; Heckel, A. Chem. Eur. J. 2015, 21, 2845. (e) Zhang, F.; Zarrine-Afsar, A.; Al-AbdulWahid, M. S.; Prosser, R. S.; Davidson, A. R.; Woolley, G. A. J. Am. Chem. Soc. 2009, 131, 2283.

(10) García-Amorós, J.; Velasco, D. Beilstein J. Org. Chem. 2012, 8, 1003.

(11) For selected examples, see: (a) Kienzler, M. A.; Reiner, A.; Trautman, E.; Yoo, S.; Trauner, D.; Isacoff, E. Y. J. Am. Chem. Soc. 2013, 135, 17683. (b) Schönberger, M.; Althaus, M.; Fronius, M.; Clauss, W.; Trauner, D. Nat. Chem. 2014, 6, 712.

(12) Klajn, R. Chem. Soc. Rev. 2014, 43, 148.

(13) (a) Frolova, S. R.; Gorbunov, V. S.; Shubina, N. S.; Perepukhov, A. M.; Romanova, S. G.; Agladze, K. I. Biosci. Rep. 2019, 39, BSR20181849. (b) Schmidt, D.; Rodat, T.; Heintze, L.; Weber, J.; Horbert, R.; Girreser, U.; Raeker, T.; Bußmann, L.; Kriegs, M.; Hartke, B.; Peifer, C. ChemMedChem 2018, 13, 2415.

(14) (a) Matsuda, K.; Higashiguchi, K. In Supramolecular Soft Matter; Nakanishi, T., Ed.; Wiley: Hoboken, 2011, 215. (b) Matsuda, K. Pure App. Chem. 2008, 80, 555.
(15) (a) Xu, W.-C.; Sun, S.; Wu, S. Angew. Chem. Int. Ed. 2019, 58, 9712. (b) Amrutha, A. S.; Sunil Kumar, K. R.; Tamaoki, N. ChemPhotoChem 2019, 3, 337.

(16) Petermayer, C.; Dube, H. Acc. Chem. Res. 2018, 51, 1153.

(17) (a) Zulfikri, H.; Koenis, M. A. J.; Lerch, M. M.; Di Donato, M.; Szymański, W.; Filippi, C.; Feringa, B. L.; Buma, W. J.J. Am. Chem. Soc. 2019, 141, 7376. (b) Lerch, M. M.; Wezenberg, S. J.; Szymanski, W.; Feringa, B. L. J. Am. Chem. Soc. 2016, 138, 6344.

(18) (a) For a review see: Crespi, S.; Simeth, N. A.; König, B. Nat. Rev. Chem. 2019, 3, 133. (b) For a selected recent example, see: Saba, S.; Dos Santos, C. R.; Zavarise, B. R.; Naujorks, A. A. S.; Franco, M. S.; Schneider, A. R.; Scheide, M. R.; Affeldt, R. F.; Rafique, J.; Braga, A. L. Chem. Eur. J. 2019, 25, in press: DOI: 10.1002/chem.201905308.

(19) For early examples of phenylazoindole dyes, see: Seferoğlu, Z; Yalçın, E.; Babür, B.; Seferoğlu, N.; Hökelek, T.; Yılmaz, E.; Şahin, E. Spectrochim. Acta, Part A 2013, 113, 314.

(20) For an early report, see: (a) Albar, H. A.; Shawali, A. S.; Abdaliah, M. A. Can. J. Chem. 1993, 71, 2144. Recently, synthesis of 3-(phenyl)diazenyl)-1,2-dimethyl-1H-indole was described as side reaction while developing base-free $\mathrm{C}-\mathrm{H}$ arylation of indoles: (b) Gemoets, H. P. L.; Kalvet, I.; Nyuchev, A. V.; Erdmann, N.; Hessel, V.; Schoenebeck, F.; Noël, T. Chem. Sci. 2017, 8, 1046.

(21) (a) Cao, D.; Zhang, Y.; Liu, C.; Wang, B.; Sun, Y.; Abdukadera, A.; Hu, H.; Liu, Q. Org. Lett. 2016, 18, 2000. (b) Liu, Y.; Ma, X.; Wu, G.; Liu, Z.; Yang, X.; Wang, B.; Liu, C.; Zhang, Y.; Huang, Y. New J. Chem. 2019, 43, 9255.

(22) Barak, D. S.; Dighe, S. U.; Avasthi, I.; Batra, S. J. Org. Chem. 2018, $83,3537$.

(23) (a) Simeth, N. A.; Crespi, S.; Fagnoni, M.; König, B. J. Am. Chem. Soc. 2018, 140, 2940. (b) Crespi, S.; Simeth, N. A.; Bellisario, A.; Fagnoni, M.; König, B. J. Phys. Chem. A 2019, 123, 1814. (c) Simeth, N. A.; Bellisario, A.; Crespi, S.; Fagnoni, M.; König, B. J. Org. Chem. 2019, 84, 6565.

(24) For an example of a synthesis of ortho-ortho'-substituted azoarenes via $\mathrm{C}-\mathrm{H}$ activation, see: (a) Hubrich, J.; Himmler, T.; Rodefeld, L.; Ackermann, L. ACS Catal. 2015, 5, 4089. (b) Himmler, T.; Rodefeld, L.; Hubrich, J.; Ackermann, L. Patent WO 2016071249 A1 20160512, 2016. 